\title{
Functional Connectivity in Healthy Subjects Is Nonlinearly Modulated by the COMT and DRD2 Polymorphisms in a Functional System-Dependent Manner
}

\author{
Tian Tian, ${ }^{1 *}$ Wen Qin, ${ }^{1 \star}$ Bing Liu, ${ }^{2,3}$ Tianzi Jiang, ${ }^{2,3,4,5}$ and Chunshui $\mathbf{Y u}^{1}$ \\ ${ }^{1}$ Department of Radiology, Tianjin Medical University General Hospital, Tianjin, China, ${ }^{2}$ Brainnetome Center and ${ }^{3}$ National Laboratory of Pattern \\ Recognition, Institute of Automation, Chinese Academy of Sciences, Beijing, China, ${ }^{4}$ Key Laboratory for NeuroInformation of Ministry of Education, School \\ of Life Science and Technology, University of Electronic Science and Technology of China, Chengdu, China, and ${ }^{5}$ Queensland Brain Institute, University of \\ Queensland, Brisbane, Queensland 4072, Australia
}

The dopamine system is known to modulate brain function in an inverted U-shaped manner. Recently, the functional networks of the brain were categorized into two systems, a "control system" and a "processing system." However, it remains unclear whether the inverted U-shaped model of dopaminergic modulation could be applied to both of these functional systems. The catechol- 0 -methyltransferase (COMT) and dopamine $\mathrm{D}_{2}$ receptor (DRD2) were genotyped in 258 healthy young human subjects. The local and long-range functional connectivity densities (FCDs) of each voxel were calculated and compared in a voxel-wise manner using a two-way (COMT and DRD2 genotypes) analysis of covariance. The resting-state functional connectivity analysis was performed to determine the functional networks to which brain regions with significant FCD differences belonged. Significant COMT $\times$ DRD2 interaction effects were found in the local FCDs of the superior portion of the right temporal pole (sTP) and left lingual gyrus (LG) and in the long-range FCDs of the right putamen and left medial prefrontal cortex (MPFC). Post hoc tests showed nonlinear relationships between the genotypic subgroups and FCD. In the control system, the sTP and putamen, components of the salience network, showed a U-shaped modulation by dopamine signaling. In the processing system, however, the MPFC of the default-mode network and the LG of the visual network showed an inverted U-shaped modulation by the dopamine system. Our findings suggest an interaction between COMT and DRD2 genotypes and show a functional system-dependent modulation of dopamine signaling.

\section{Introduction}

Many psychiatric diseases, including schizophrenia, depression, and autism, have been associated with dysfunction of the dopamine system, the function of which is modulated by genetic variations (Robinson et al., 2001; Witte and Flöel, 2012). The dopamine system modulates structure and function of the brain in a nonlinear manner that has been commonly described as an inverted U-shaped relationship (Williams and Goldman-Rakic, 1995; Goldman-Rakic, 1998; Seamans and Yang, 2004; Bertolino et al., 2009a). The functional networks of the human brain have been categorized into two independent functional systems: the "processing system" includes the visual, sensorimotor, and

Received May 21, 2013; revised Aug. 12, 2013; accepted Sept. 26, 2013.

Author contributions: T.T., W.Q., T.J., and C.Y. designed research; T.T. and W.Q. performed research; T.T., W.Q., and B.L. contributed unpublished reagents/analytic tools; T.T., W.Q., and B.L. analyzed data; T.T. and C.Y. wrote the paper.

The authors declare no competing financial interests.

This work was supported by the National Basic Research Program of China (973 program, Grant 2011CB707800), the Natural Science Foundation of China (Grants 81271551 and 91132301), and the Strategic Priority Research Program of the Chinese Academy of Sciences (Grant XDB02030300).

*T.T. and W.Q. contributed equally to this work.

Correspondence should be addressed to Dr. Chunshui Yu, Department of Radiology, Tianjin Medical University General Hospital, No. 154, Anshan Road, Heping District, Tianjin 300052, China. E-mail: chunshuiyu@tijmu.edu.cn. DOI:10.1523/JNEUROSCI.2163-13.2013

Copyright $\odot 2013$ the authors $\quad 0270-6474 / 13 / 3317519-08 \$ 15.00 / 0$ default-mode networks, and the "control system" consists of the fronto-parietal, attention, and salience networks (Power et al., 2011). However, it remains unclear whether the inverted U-shaped model of dopaminergic modulation can be applied to both functional systems.

Catechol-O-methyltransferase (COMT) catalyzes the degradation of synaptic dopamine in the brain, especially in the prefrontal cortex (PFC) because of the lack of dopamine transporter in PFC synapses (Männistöand Kaakkola, 1999; Seamans and Yang, 2004). The COMT gene contains a functional polymorphism (Val158Met), resulting in a fourfold decrease in enzymatic activity at body temperature in Met-allele carriers (Männistöand Kaakkola, 1999). This decrease in enzymatic activity leads to increased synaptic dopamine concentration that further affects structure and function of the brain. Dopamine $\mathrm{D}_{2}$ receptor (DRD2) has two alternatively spliced isoforms (Khan et al., 1998; Usiello et al., 2000), the ratio of which is modulated by a DRD2 gene polymorphism (rs1076560, G>T) and further regulates dopamine signaling in both healthy subjects (Zhang et al., 2007) and patients with schizophrenia (Bertolino et al., 2009b). Different genotypic combinations of these two single-nucleotide polymorphisms (SNPs) may generate subgroups of subjects with different levels of dopamine signaling; these subgroups can be used to investigate the nonlinear modulatory patterns of the dopamine system. The inverted U-shaped modulation of COMT or DRD2 
genetic variant on behavioral performance (Mattay et al., 2003; Giakoumaki et al., 2008; Fallon et al., 2013) or brain function (Bertolino et al., 2009a; Dang et al., 2012) has been reported separately. Based on the interaction effects between COMT and DRD2 on behavioral performance (Reuter et al., 2006; Xu et al., 2007), we hypothesize that brain functional organization may also show interaction effects between COMT and DRD2.

The functional connectivity density (FCD) mapping is a newly developed data-driven method to identify the distribution of energy-efficient hubs in the human brain (Tomasi and Volkow, 2010, 2011a,b). In this study, we used the FCD approach in healthy young subjects to explore the impact of COMT Val158Met and DRD2 rs1076560 functional polymorphisms on the FCDs throughout the whole brain. We hypothesize that the dopamine system may exhibit different nonlinear modulation on the FCDs of the control and processing functional systems because of their independent or anticorrelated functionalities.

\section{Materials and Methods}

Subjects. A total of 306 right-handed healthy young adults were recruited for this study. Participants were carefully screened to ensure that they had no history of psychiatric or neurological illness, psychiatric treatment, or drug or alcohol abuse and that they had no contraindications to MRI examination. Only Chinese Han populations were included to purify the sample. All subjects were strongly right-handed according to the Chinese edition of the Edinburgh Handedness Inventory (Oldfield, 1971). The study was approved by the Medical Research Ethics Committee of Tianjin Medical University, and all participants provided written informed consent. Memory function was assessed using the Chinese Revised Wechsler Memory Scale (Gong, 1989), and executive function was assessed using the Wisconsin Card Sorting Test (Heaton, 1999). Individual working memory capacity was evaluated with the n-back task (Owen et al., 2005). Depression levels were evaluated with the Beck Depression Inventory (Beck and Steer, 1993), and anxiety levels were examined using the Self-Rating Anxiety Scale (Zung, 1971). Temperamental characteristics were tested using the Tridimensional Personality Questionnaire (Cloninger et al., 1993). Forty-eight subjects were excluded from further analysis because of poor imaging quality ( 22 subjects) or genotyping failure ( 26 subjects). The remaining 258 healthy young adults ( $141 \mathrm{fe}-$ males and 117 males; mean age, $22.8 \pm 2.4$ years; range, $18-29$ years) were ultimately included in the imaging analysis.

Genotyping. We extracted genomic DNA from $3000 \mu$ l of whole blood using the EZgeneTM Blood gDNA Miniprep kit (Biomiga). We then determined each subject's genotypes for COMT rs4680 and DRD2 rs 1076560 using the PCR and ligation detection reaction (LDR) method (Thomas et al., 2004; Yi et al., 2009) with technical support from the Shanghai Biowing Applied Biotechnology Company. The PCR primer sequences for COMT were as follows: forward, 5' GGGCCTACTGTGGCTACTCA 3'; reverse, 5' CCCTTTTTCCAGGTCTGACA 3'. The PCR primer sequences for DRD2 were as follows: forward, 5' AGCATCTCCATCTCCAGCTC 3'; reverse, 5' GAAAAAGGACAGGGGCAATC 3'. PCR was performed with a $20 \mu \mathrm{l}$ reaction volume containing $1 \mu \mathrm{l}$ of genomic DNA, $0.4 \mu \mathrm{l}$ of primer mixture, $2 \mu \mathrm{l}$ of dNTPs, $0.6 \mu \mathrm{l}$ of $\mathrm{Mg}^{2+}$, $2 \mu \mathrm{l}$ of buffer, $4 \mu \mathrm{l}$ of Q-Solution, and $0.3 \mu \mathrm{l}$ of TaqDNA polymerase. The amplification protocol consisted of an initial denaturation and enzyme activation phase at $95^{\circ} \mathrm{C}$ for $15 \mathrm{~min}$, followed by 35 cycles of denaturation at $94^{\circ} \mathrm{C}$ for $30 \mathrm{~s}$, annealing for $1 \mathrm{~min}$ and $30 \mathrm{~s}$ at $59^{\circ} \mathrm{C}$ for COMT rs 4680 and $56^{\circ} \mathrm{C}$ for DRD2 rs 1076560 , extension at $72^{\circ} \mathrm{C}$ for $1 \mathrm{~min}$, and a final extension at $72^{\circ} \mathrm{C}$ for $7 \mathrm{~min}$. PCR products were verified in $3 \%$ agarose gels that had been stained with ethidium bromide to regulate the amount of DNA added to the LDR.

For each SNP, three probes were designed for the LDRs: one common probe (rs4680, P-GCCAGCGAAATCCACCATCCGCTGGTTTTTTTT TTTTTTTTTTTT-FA; rs1076560, P-GAAAGGGAGGGGCCAGTGAGATGGGTTTTTTTTTTTTTTTTTT-FAM) and two discriminating probes for the two alleles of each SNP (rs4680_A, TTTTTTTTTTT TTTTTTTTTCAGGCATGCACACCTTGTCCTTCAT; rs4680_G, TTTTT
TTTTTTTTTTTTTTTTTCAGGCATGCACACCTTGTCCTTCAC; rs1076560_T, TTTTTTTTTTTTTTTTTTGTGTTTGCAGGAGTCT TCAGAGGGT; rs1076560_G, TTTTTTTTTTTTTTTTTTTTGTGTTTGCAGGAGTCTTCAGAGGGG). These reactions were conducted in a $10 \mu \mathrm{l}$ mixture containing $1 \mu \mathrm{l}$ of buffer, $1 \mu \mathrm{l}$ of probe mix, $0.05 \mu \mathrm{l}$ of TaqDNA ligase, $1 \mu \mathrm{l}$ of PCR product, and $6.95 \mu \mathrm{l}$ of deionized water. The reaction program consisted of an initial heating at $95^{\circ} \mathrm{C}$ for $2 \mathrm{~min}$, followed by 35 cycles of $30 \mathrm{~s}$ at $94^{\circ} \mathrm{C}$ and $2 \mathrm{~min}$ at $50^{\circ} \mathrm{C}$. Reactions were stopped by chilling the tubes in an ethanol-dry ice bath and adding $0.5 \mathrm{ml}$ of $0.5 \mathrm{~mm}$ EDTA. Aliquots of the reaction products $(1 \mu \mathrm{l})$ were mixed with $1 \mu \mathrm{l}$ of loading buffer ( $83 \%$ formamide, 8.3 mm EDTA, and $0.17 \%$ blue dextran) and $1 \mu$ lof ABI GS-500 Rox-Fluorescent molecular weight marker and denatured at $95^{\circ} \mathrm{C}$ for $2 \mathrm{~min}$. The samples were then chilled rapidly on ice before being loaded on a $5 \mathrm{M}$ urea-5\% polyacrylamide gel and electrophoresed on an ABI 3100 DNA Sequencer at 3000 V. Finally, the fluorescent ligation products were analyzed and quantified using the ABI GeneMapper software.

Image acquisition. MR images were acquired using a Signa HDx 3.0 tesla MR scanner (General Electric). Tight but comfortable foam padding was used to minimize head motion, and ear plugs were used to reduce scanner noise. Resting-state fMRI data were obtained using single-shot echoplanar imaging with the following parameters: repetition time (TR)/echo time (TE), 2000/30 ms; field of view (FOV), $240 \times$ $240 \mathrm{~mm}$; matrix, $64 \times 64$; flip angle (FA), $90^{\circ}$; slice thickness, $4 \mathrm{~mm}$; no gap; 40 interleaved transverse slices; 180 volumes. During the fMRI scans, all subjects were instructed to keep their eyes closed, to relax and move as little as possible, to think of nothing in particular, and to not fall asleep. To better coregister the fMRI data, sagittal 3D T1-weighted images were acquired using a brain volume (BRAVO) sequence (TR/TE, 8.1/3.1 ms; inversion time, $450 \mathrm{~ms}$; FA, $13^{\circ}$; FOV $256 \times 256 \mathrm{~mm}$; matrix, $256 \times 256$; slice thickness, $1 \mathrm{~mm}$; no gap; 176 sagittal slices).

Data preprocessing. The resting-state fMRI data were preprocessed using SPM8 (http://www.fil.ion.ucl.ac.uk/spm). The first 10 volumes for each subject were discarded to allow the signal to reach equilibrium and the participants to adapt to the scanning noise. The remaining $170 \mathrm{vol}-$ umes were then corrected for the acquisition time delay between slices. All subjects' fMRI data were within the defined motion thresholds (translational or rotational motion parameters lower than $2 \mathrm{~mm}$ or $2^{\circ}$ ). We also calculated framewise displacement (FD), which indexes volume-tovolume changes in head position. These changes were obtained from the derivatives of the rigid-body realignment estimates that are used to realign blood oxygen level-dependent (BOLD) data during fMRI preprocessing (Power et al., 2012,2013). There was no main effect of each SNP and interaction on the FD ( $p>0.05)$. The approach used to normalize these functional images included the following steps: (1) individual structural images were linear coregistered to the mean functional image after motion correction; (2) the transformed structural images were segmented into gray matter, white matter, and CSF, and gray matter was nonlinear coregistered to the Montreal Neurological Institute (MNI) space; and (3) the motion-corrected functional volumes were spatially normalized to the MNI space using the parameters estimated during nonlinear coregistration. The functional images were then resampled into a voxel size of $3 \times 3 \times 3 \mathrm{~mm}^{3}$. After normalization, the datasets were bandpass filtered with frequency from 0.01 to $0.1 \mathrm{~Hz}$, and several nuisance covariates (six motion parameters and average BOLD signals of the ventricular and white matter) were regressed out from the data.

Most of the data preprocessing steps for the region of interest (ROI)based resting-state functional connectivity ( $\mathrm{rsFC}$ ) analysis were the same as the preprocessing steps for the FCD calculation. The differences included following steps: (1) after normalization, images were smoothed using a Gaussian kernel of $8 \times 8 \times 8 \mathrm{~mm}^{3}$ full-width at half-maximum; and (2) the average BOLD signals of the whole brain were regressed out from the data for displaying anticorrelated networks (Fox et al., 2005).

FCD calculation. We calculated the FCD of each voxel using the inhouse script that was written in the Linux platform according to the method described by Tomasi and Volkow (2010). The Pearson's linear correlation was used to calculate the functional connections, and two voxels with a correlation coefficient of $R>0.6$ were considered function- 


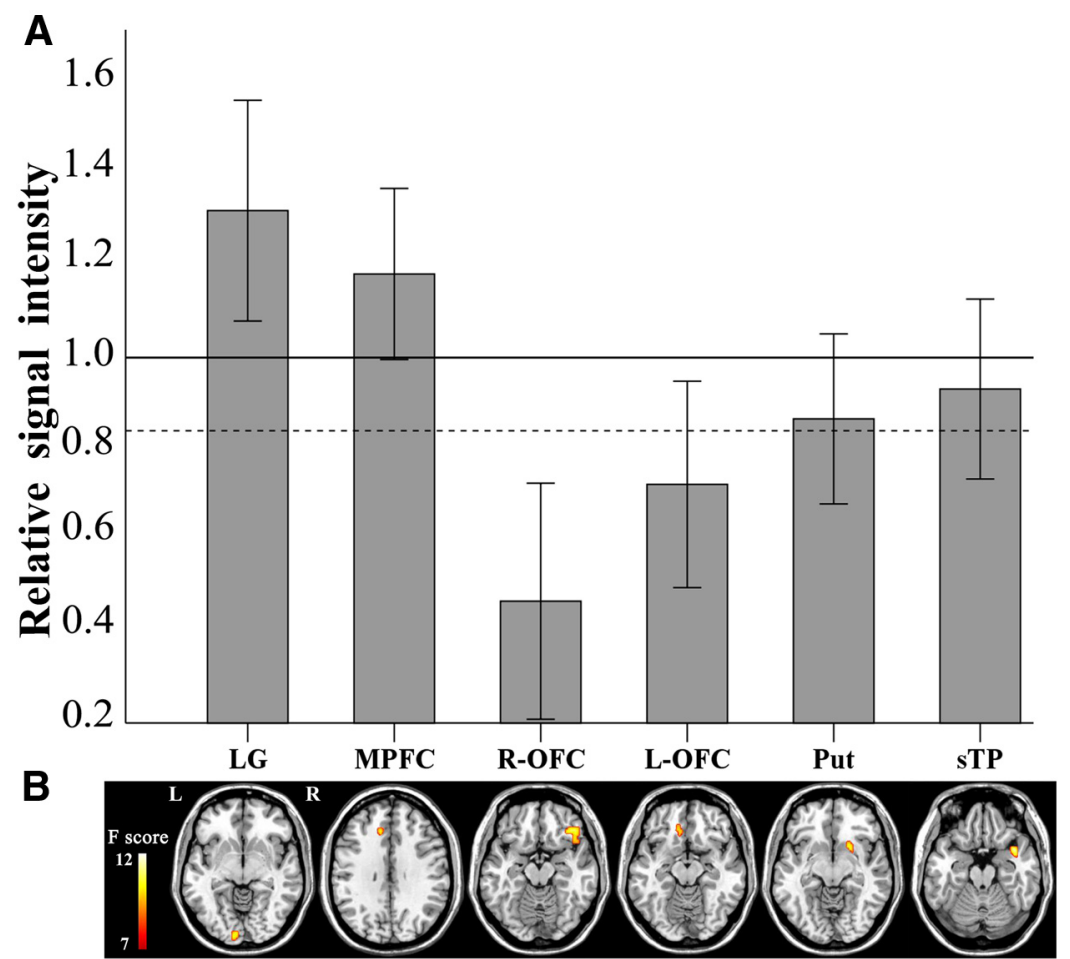

Figure 1. Relative MRI signal intensities $(\boldsymbol{A})$ of brain regions $(\boldsymbol{B})$ with significant main effects of genotypes or interactions between genotypes on local or long-range FCDs. The horizontal axis represents six brain regions with significant main effects of genotypes or interactions between genotypes. The solid line represents the mean signal intensity of the whole-brain gray matter. The dashed line represents the lower limit of twice the SD of the signal intensity of the whole-brain gray matter. Bars show the $F$ values. The two orbitofrontal clusters were excluded from additional analyses because the mean signal intensities of the two clusters were lower than the lower limit. L, Left; L-OFC, left orbitofrontal cortex; Put, putamen; R, right; R-OFC, right orbitofrontal cortex.

Table 1. Demographic data of subjects in $\mathrm{fMRI}$ analysis $(n=258)$

\begin{tabular}{lrlll}
\hline Genotypic groups & $n$ & Age (years) & $\begin{array}{l}\text { Years of } \\
\text { education }\end{array}$ & $\begin{array}{l}\text { Gender } \\
\text { (males/females) }\end{array}$ \\
\hline $\begin{array}{l}\text { COMT } \\
\text { Val/Val }\end{array}$ & 126 & $22.9(2.5)$ & $15.9(2.1)$ & $55: 71$ \\
$\quad$ Met carriers & 132 & $22.7(2.3)$ & $15.5(2.2)$ & $62: 70$ \\
$\quad F(P)$ & 258 & $0.34(0.56)$ & $1.37(0.24)$ & $0.76(0.38)$ \\
DRD2 & & & & \\
TT & 42 & $22.7(2.8)$ & $15.7(2.1)$ & $16: 26$ \\
GT & 118 & $22.9(2.3)$ & $15.8(2.2)$ & $54: 64$ \\
GG & 98 & $22.7(2.3)$ & $15.6(2.0)$ & $47: 51$ \\
$F(P)$ & 258 & $0.15(0.87)$ & $0.04(0.97)$ & $0.46(0.63)$ \\
COMT $\times$ DRD2 & & & & \\
Val/Val-TT & 23 & $22.7(2.7)$ & $15.7(1.9)$ & $7: 16$ \\
Val/Val-GT & 59 & $22.6(2.5)$ & $15.8(2.2)$ & $28: 31$ \\
Val/Val-GG & 44 & $23.3(2.3)$ & $16.1(2.0)$ & $20: 24$ \\
Met carriers-TT & 19 & $22.7(3.0)$ & $15.7(2.4)$ & $9: 10$ \\
Met carriers-GT & 59 & $23.1(2.1)$ & $15.7(2.3)$ & $26: 33$ \\
Met carriers-GG & 54 & $22.2(2.2)$ & $15.3(2.0)$ & $27: 27$ \\
$F(P)$ & 258 & $3.26(0.04)$ & $0.96(0.39)$ & $0.65(0.52)$ \\
\hline
\end{tabular}

ally connected. The calculation of the FCD was restricted to voxels in the gray matter regions with a signal-to-noise ratio of $>50 \%$ to minimize unwanted effects from susceptibility-related signal-loss artifacts (Tomasi and Volkow, 2010). The local FCD (IFCD) at a given voxel $x_{0}$ was computed as the local $k\left(x_{0}\right)$ between $x_{0}$ and its neighbor voxels using a "growing" algorithm developed in the Linux platform. Specifically, a voxel $\left(x_{j}\right)$ was added to the list of voxels functionally connected with $x_{0}$ only if it was adjacent to a voxel that was linked to $x_{0}$ by a continuous path of functionally connected voxels and $R_{0 \mathrm{j}}>0.6$ (Tomasi and Volkow, 2010). This calculation was repeated for all voxels that were adjacent to voxels that are included in the list of voxel functionally connected to $x_{0}$ in an iterative manner until no new voxels could be added to the list. The local FCD at $x_{0}$ was computed as the number of elements in the local functional connectivity cluster, $k\left(x_{0}\right)$. Then, the calculation was initiated for a different $x_{0}$ and was finally applied to all qualified voxels of the brain (Tomasi and Volkow, 2010). The global FCD (gFCD) at a given voxel $x_{0}$ was computed as the global number of functional connections, $k\left(x_{0}\right)$, between $x_{0}$ and all other voxels. This calculation was repeated for all $x_{0}$ voxels in the brain. The strength of the longrange FCD was equated to gFCD - IFCD to remove all connected voxels that belonged to the local cluster (Tomasi and Volkow, 2010). The Kolmogorov-Smirnov test was applied to determine whether the local and long-range FCD data satisfy normal distributions. We found that both the local $(z=3.45, p<0.001)$ and long-range ( $z=1.66, p=0.008)$ FCD values did not conform to normal probability distribution. To increase the normality of the distribution, grand mean scaling of local and long-range FCDs was performed by dividing by the mean value of the qualified voxels of the whole brain. After grand mean scaling, both the local $(z=1.20, p=0.113)$ and long-range $(z=0.92, p=0.368)$ FCD values conformed to normal probability distribution and were suitable for the parametric statistical inference. Finally, the normalized FCDs were spatially smoothed with an $8 \times 8 \times 8 \mathrm{~mm}^{3}$ Gaussian kernel.

Statistical analysis. Statistical analyses for demographic, cognitive, and psychological data were performed using the Statistical Package for the Social Sciences version 18.0 (SPSS) for Windows. A two-way (COMT genotype and DRD2 genotype) ANOVA was used to evaluate the main effects of each SNP and their interactions for demographic, cognitive, and psychological data. We were interested in both the main effects of each SNP and their interactions.

The voxel-wise comparisons of FCD mapping were performed using a two-way (COMT genotype and DRD2 genotype) ANOVA with the age as a nuisance variable. Several clusters showed significant COMT $\times$ DRD2 interaction effects (uncorrected $p<0.001$; cluster size, $>30$ voxels across the whole brain) on FCDs. However, these clusters could not survive after a whole-brain family-wise error (FWE) correction $(p<0.05)$ for multiple comparisons. Since a whole-brain FWE correction is a rather conservative method, we then applied a small-volume correction to reduce the number of comparisons. For each of these significant clusters on the basis of the whole-brain findings, a 20 -mm-radius sphere centered at the peak location of the cluster was placed around each cluster, and the FWE small-volume correction $(p<0.05)$ was used to correct for multiple comparisons within the sphere. To exclude the influence of signal loss on the results, we extracted the signal intensities of these regions and computed the ratio between the mean signal of each region and that of the whole-brain gray matter (Fig. 1). Two orbitofrontal regions were excluded from additional analysis because of a much lower signal intensity compared with the whole-brain gray matter.

To determine the functional networks to which brain regions with significant differences in FCDs belonged, we defined these regions as ROIs for the whole-brain rsFC analysis. On the basis of the rsFC patterns of these ROIs, we inferred the possible network to which each ROI belonged. Then we reconstructed each presumed network using an independent ROI-based rsFC analysis. Each seed ROI was defined as 6-mmdiameter spheres around a previously identified peak coordinate of the cluster, i.e., the primary visual cortex (peak MNI coordinates: $x=-18$, 

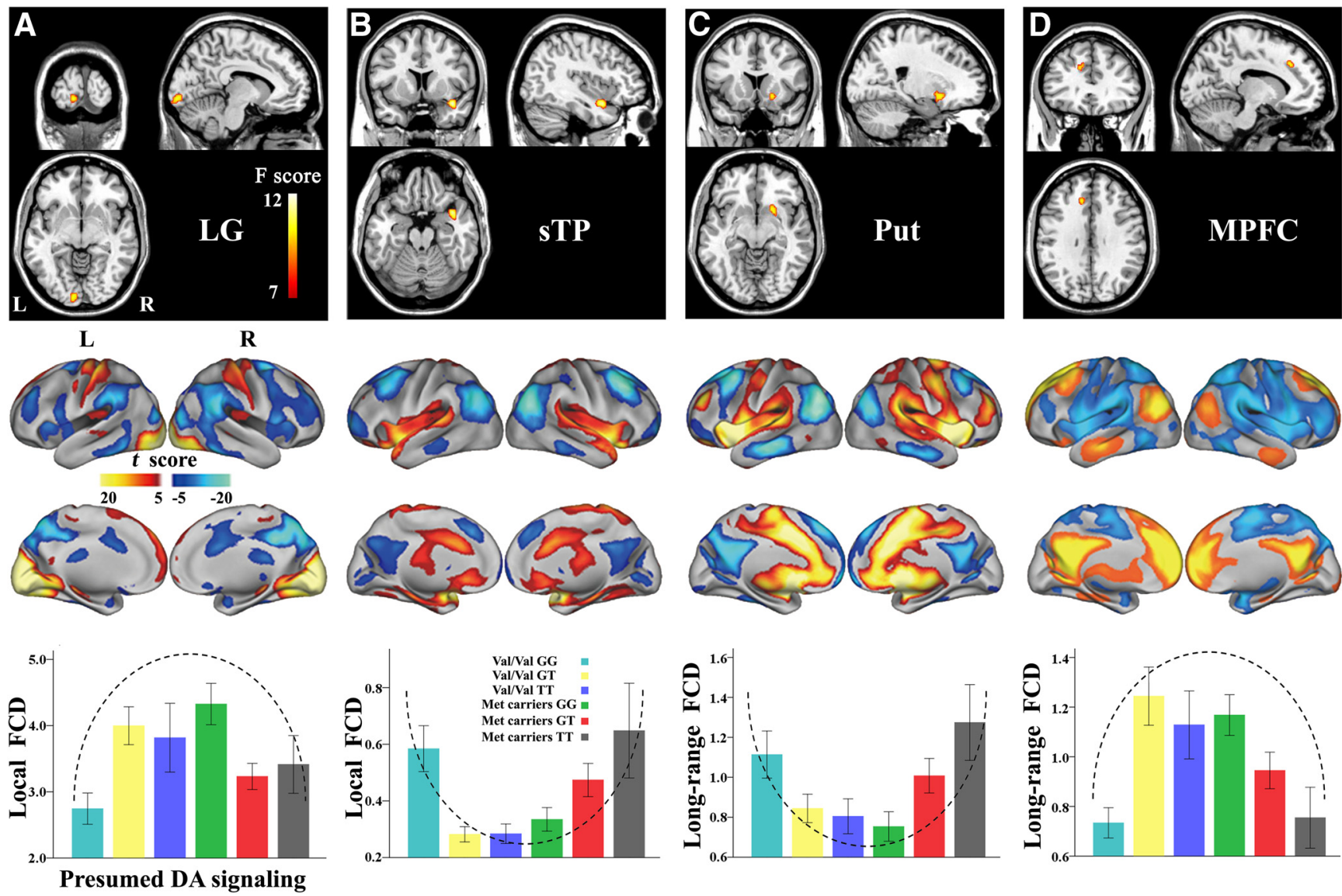

Figure 2. Brain regions with significant COMT $\times$ DRD2 interaction effects on FCDs (top row), the rsFC maps of these significant clusters (middle row), and the modulation effect of the presumed dopamine signaling on the FCD of each cluster (bottom row) are shown. $\boldsymbol{A}-\boldsymbol{D}$ represent the four significant clusters. Colored bars show the $F$ or $t$ values. The horizontal axes of the bar plots (bottom row) represent six genotypic subgroups with presumed dopamine availability from low to high. The dashed lines represent the hypothesized dopamine modulation patterns (U shape or inverted $U$ shape). DA, Dopamine; L, left; Put, putamen; R, right.

\begin{abstract}
$y=-96, z=1$ ) for the visual network (Büchel and Friston, 1997), the posterior cingulate cortex $(x=-3, y=-48, z=30)$ for the defaultmode network (DMN) (Liu et al., 2010), and the right anterior insula $(x=36, y=16, z=4)$ for the salience network (Dosenbach et al., 2007). After reconstructing these functional networks, we projected each significant cluster onto the mask of the presumed functional network to determine whether the cluster was located in the network. The ROI-based whole-brain rsFC analyses were performed as follows: (1) for each subject, the correlation coefficient between the mean time series of each ROI and that of each voxel in the whole brain was computed and converted into a $z$ value to improve normality using the Fisher's $r$-to- $z$ transformation; and (2) individuals' $z$ values were entered into a random-effect one-sample $t$ test to identify the brain regions that were significantly correlated with the ROI. The significant rsFC maps were corrected for multiple comparisons using the FWE $(p<0.05)$ method.
\end{abstract}

\section{Results}

Demographic and genetic characteristics

Detailed demographic data are summarized in Table 1 . The distributions of COMT rs4680 genotypes (126 Val/Val, 106 $\mathrm{Met} / \mathrm{Val}$, and $26 \mathrm{Met} / \mathrm{Met}$ ) and DRD2 rs1076560 genotypes (42 TT, 118 GT, and 98 GG) were both in Hardy-Weinberg equilibrium $(p>0.05)$. Subjects who were either homozygous or heterozygous for the Met-allele of COMT were merged into a group of Met-allele carriers because of the relatively low frequency of Met homozygotes (four to five times lower than Val homozygotes); this method has been used previously to address skewed genotypic distributions (Taylor et al., 2007;
Aguilera et al., 2008; Ettinger et al., 2008; Li et al., 2009). A significant interaction between SNPs was found for age $(p<$ 0.05 ) but not for years of education or gender. No significant main effects were found for any demographic variables $(p>$ $0.05)$. Neither significant main effects of any SNPs nor significant interactions between the two SNPs $(p>0.05)$ were found for any of these cognitive (memory and execution) and psychological (depression, anxiety, and personality) variables, suggesting that these genotypic subgroups are matched in these neuropsychological variables.

\section{FCD analysis}

Although neither of the two SNPs showed a significant main effect, significant interaction effects (uncorrected $p<0.001$; cluster size, $>30$ voxels across the whole brain) were found between the COMT and DRD2 genotypes in the local FCDs of the left lingual gyrus (LG) (Fig. 2A, top row) and the superior portion of the right temporal pole (sTP) (Fig. $2 B$, top row) (Table 2). After applying an FWE small-volume correction for multiple comparisons $(p<0.05)$, the local FCDs of both the left LG $(z=3.72 ; p=$ $0.019)$ and the $\operatorname{sTP}(z=4.20 ; p=0.003)$ were still significant (Table 2). The mean local FCD values of these two clusters were extracted from each subject. For each genotypic subgroup (Val/Val-GG, Val/Val-GT, Val/Val-TT, Met carriers-GG, Met carriers-GT, and Met carriers-TT), the means and SEs of the local FCDs of the two clusters are shown in the bottom rows of Figure 
Table 2. Brain areas showed significant COMT $\times$ DRD2 interaction effects on FCDs

\begin{tabular}{lllllrr}
\hline FCD & $\begin{array}{l}\text { Brain } \\
\text { regions }\end{array}$ & $\begin{array}{l}\text { Brodmann } \\
\text { areas }\end{array}$ & $\begin{array}{l}\text { Cluster } \\
\text { size (voxels) }\end{array}$ & $\begin{array}{l}\text { MNI coordinates } \\
(x, y, z)\end{array}$ & $\begin{array}{l}\text { Peak } F \\
\text { values }\end{array}$ & \begin{tabular}{r} 
FWE small-volume correction \\
\cline { 5 - 8 }
\end{tabular} \\
\hline Local FCD & Left LG & 17,18 & 35 & $-9,-93,-6$ & 9.57 & 3.72 \\
Local FCD & Right sTP & 38 & 40 & $39,9,-18$ & 11.76 & 0.20 \\
Long-range FCD & Right putamen & & 31 & $21,12,-9$ & 9.35 & 3.67 \\
Long-range FCD & Left MPFC & 9,32 & 31 & $-15,30,33$ & 9.46 & 3.70 \\
\hline
\end{tabular}

The peak F values represent the statistical results of the voxel-based FCD analyses. Statistical results after FWE small-volume correction are shown in the last two columns.
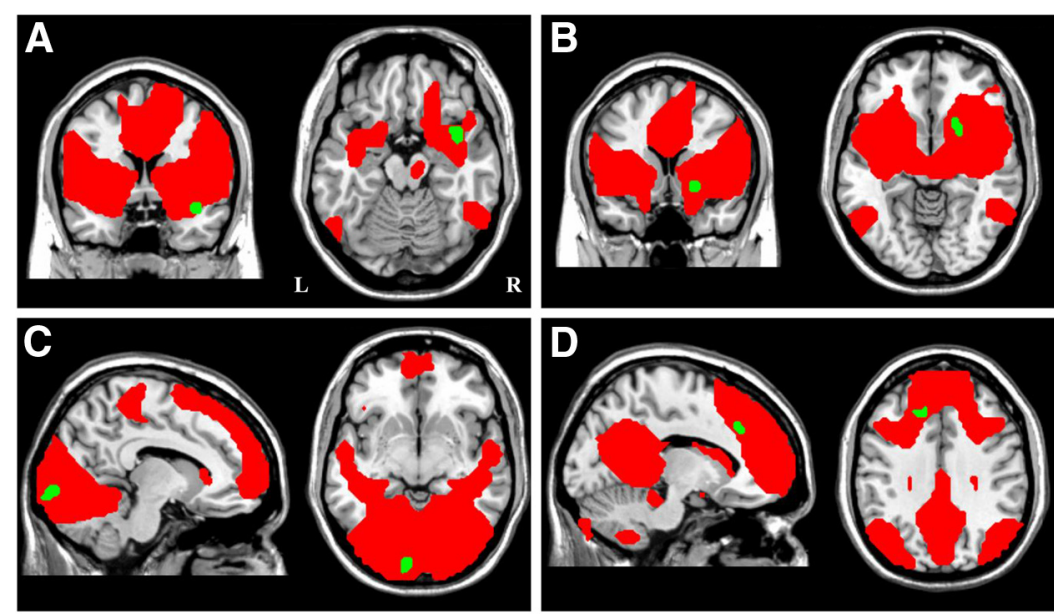

Figure 3. Overlaps of the significant clusters (green) of the $\operatorname{STP}(\boldsymbol{A})$, the putamen $(\boldsymbol{B})$, the LG $(\boldsymbol{C})$, and the MPFC (D) with their corresponding functional network masks (red). The red mask represents the salience network $(\boldsymbol{A}, \boldsymbol{B})$, the visual network $(\boldsymbol{C})$, and the $\operatorname{DMN}(\boldsymbol{D})$. L, Left; R, right.

2, $A$ and $B$. The distribution of the local FCD of these genotypic subgroups (which reflect different levels of presumed dopamine signaling) was more likely an inverted $\mathrm{U}$ shape in the LG (Fig. $2 A$, bottom row) and a $U$ shape in the right sTP (Fig. $2 B$, bottom row).

Significant interaction effects were also found between the COMT and DRD2 in the long-range FCDs of the right putamen $(z=3.67 ; p=0.037$ FWE small-volume correction) (Fig. 2C, top row $)$ and left medial prefrontal cortex (MPFC) $(\mathrm{z}=3.70 ; p=$ 0.034 FWE small-volume correction) (Fig. 2D, top row). Additional analysis showed that the distribution of the long-range FCD of these genotypic subgroups was more likely a $U$ shape in the right putamen (Fig. 2C, bottom row) and an inverted $U$ shape in the MPFC (Fig. 2D, bottom row).

It has been reported that the FCD showed gender differences (Tomasi and Volkow, 2012). To test potential gender effects on the FCD analysis, we extracted the FCDs of these four significant clusters and performed a full factorial ANOVA with gender, COMT genotype, and DRD2 genotype as factors. We did not find either any significant main effects of gender or any significant interactions of gender $\times$ COMT, gender $\times$ DRD2, and gender $\times$ COMT $\times$ DRD2 $(p>0.05)$, suggesting that the gender did not significantly influence our findings.

The rsFC pattern of each significant cluster is shown in Figure 2 (middle row), and the overlap between each significant cluster and its corresponding functional network is shown in Figure 3. We found that the sTP and putamen were both components of the salience network, that the MPFC was a part of the DMN, and that the LG was a part of the visual network.

\section{Discussion}

In the present study, we investigated the modulation of two dopamine SNPs (COMT rs4680 and DRD2 rs1076560) on the FCDs in healthy young adults. We found completely different nonlinear modulation patterns of the dopamine system on the FCDs of the different functional systems. In the control system, the sTP and putamen, components of the salience network, showed a U-shaped modulation. In the processing system, however, the MPFC of the DMN and the LG of the visual network showed an inverted U-shaped modulation.

The exact neural mechanisms by which dopamine SNPs affect brain functional connectivity are still unclear. A candidate for this neural mechanism may be the dopamine level-dependent neurotrophic and neurotoxic effects (Honea et al., 2009). Consistent with the nonlinear relationship between the dopamine levels and neuronal activity (Williams and Goldman-Rakic, 1995; Seamans and Yang, 2004; Bertolino et al., 2009b; Qin et al., 2012), the effect of dopamine levels on neuronal survival and growth has been described as an inverted U-shaped curve. In this model, an optimal extracellular dopamine level may induce the generation of brain derived neurotrophic factor (BDNF) (Kuppers and Beyer, 2001) and facilitate neuronal growth; however, both lower and higher extracellular dopamine levels may impair neuronal integrity and survival (Santiago et al., 2000). For example, excessive extracellular dopamine level in dopamine transporter knockout mice can reduce BDNF gene expression in frontal cortex (Fumagalli et al., 2003). In contrast, reduced dopamine signaling in D1 receptor mutant mice can impair the expression of dopamine-mediated behavioral responses by affecting the neurochemical architecture of the striatum (Xu et al., 1994). Pharmacological studies in both animals (Granon et al., 2000) and humans (Kimberg et al., 1997; Mattay et al., 2000; Mehta et al., 2000; Mattay et al., 2003; Apud et al., 2007) reported that poor cognitive performance of individuals with low dopamine levels tended to be improved by dopaminergic stimulants, whereas the performance of individuals who have dopamine levels near or at the top of inverted U-shaped curve showed no improvement or even deterioration. These studies suggest that the effect of dopamimetic agents on the PFC functioning depends on the baseline dopamine levels and the relative location on the inverted $\mathrm{U}$-shaped curve.

Although the inverted U-shaped relationship between dopamine signaling and neuronal activity has been repeatedly shown, it is still unclear whether this inverted U-shaped model of dopaminergic modulation could be applied to different functional systems. The human brain has been categorized into two independent functional systems: the processing system includes the DMN and visual and sensorimotor networks; and the control system consists of the salience, attention, and fronto-parietal networks (Power et al., 2011). This framework makes it possible to 
explore if the effects of the dopamine system on the brain are in a functional-system-dependent manner.

In the processing system, we found an inverted U-shaped modulation of dopamine signaling on the FCDs of the MPFC within the DMN and the LG of the visual network. As a critical node within the DMN, the MPFC is confirmed to be modulated by COMT or DRD2 in human imaging genetic studies (Liu et al., 2010; Sambataro et al., 2011). Consistent with our findings, an earlier study has revealed an inverted U-shaped relationship between the dopamine level and the prefrontal rsFCs of the DMN (Dang et al., 2012). However, the LG, as a node of the visual network, has rarely been associated with the dopamine system. Thus, the functional significance of the inverted U-shaped modulation of dopamine signaling on the FCDs of the LG needs to be clarified in future studies.

In the control system, we found a U-shaped modulation of dopamine signaling on the FCDs of brain regions (the sTP and putamen) of the salience network. The salience network serves to identify salient stimuli and to initiate transient control signals to modulate higher-order cognitive processes (Sridharan et al., 2008; Menon and Uddin, 2010). The dopamine system plays a crucial role in the physiology of the salience network (Palaniyappan and Liddle, 2012), with an inverted U-shaped modulation on both memory task-evoked activation and grey matter volumes of brain regions belonging to the salience network (Bertolino et al., 2009b; Qin et al., 2012). The putamen is a critical component of the striatum, whose function and structure are both modulated by dopamine signaling in an inverted $\mathrm{U}$-shaped manner (Bertolino et al., 2009b; Wallace et al., 2011). Although less attention has been paid to the sTP of the salience network, both reduced temporal lobe volumes and elevated DRD2 densities are shown in patients with chronic schizophrenia (Tune et al., 1996). Moreover, the amphetamine-induced striatal dopamine release may induce hyperactivity in temporal lobe and putamen (O'Daly et al., 2011). It seems that a U-shaped modulation of dopamine signaling on the salience network is inconsistent with the inverted U-shaped theory of cognitive control of the dopamine system (Williams and Goldman-Rakic, 1995; Vijayraghavan et al., 2007; Bertolino et al., 2009b; Cools and D'Esposito, 2011). A possible reason may be the modulation of dopamine system on brain function being dependent on functional measures. For example, in a reversal learning task, the activation of the salience network regions followed an inverted U-shaped pattern; in contrast, the fronto-striatal connectivity followed a U-shaped pattern (Cohen et al., 2007). Moreover, both the inverted and upright U-shaped dopamine modulations on structural profiles of the salience network and DMN have been shown in previous studies (Bertolino et al., 2009b; Honea et al., 2009).

One of the most important findings of this study is the inverse modulation patterns of dopamine system on the FCDs of the processing system and the control system (Fig. 2). This finding is consistent with the modulation pattern of catecholaminergic signaling on stress-induced activation during the working memory task. In that study, the DMN of the processing system exhibited a U-shaped modulation, whereas the dorsal fronto-parietal network of the control system demonstrated an inverted U-shaped modulation (Qin et al., 2012). This inversed modulation of the dopamine system on brain function may be associated with anticorrelated relationship between the processing and control systems in the human brain (Power et al., 2011). Although the mechanisms of the functional network-dependent modulation of the dopamine system are not clear, a balance mechanism may possibly explain the phenomenon. The processing system is thought to be relatively stationary, whereas behavioral control mechanisms in the control system have more dynamic task sets and must flexibly adapt processing to a wide range of tasks (Posner and Petersen, 1990; Power et al., 2011). Individuals with Val/ Val-TT or Met-GG genotype have optimal dopamine levels and show higher FCDs in brain regions of the processing system; the highly connected processing system may need less modulation from the control system. The lack of demands for cognitive control may be related to the sparse connections in the control system in these subjects. In contrast, individuals with Val/Val-GG and Met-TT genotype have dopamine levels that are either too low or too high and show lower FCDs in brain regions of the processing system. The sparsely connected processing system needs more modulation from the control system to complete behaviors in a normal manner, compared with the modulation system utilized by the other genotypic subjects. The enhanced demands for cognitive control may result in dense connections in the control system. This hypothesis may explain why different genotypic subgroups did not show significant differences in any of the cognitive, emotional, or personality scores. However, it should be noted that the DRD2 is much more important in the striatal dopamine modulation (Sambataro et al., 2011), whereas the COMT is specifically related to the PFC dopamine modulation (Akil et al., 2003). Moreover, the reciprocal relationship between these regions is rather complex. Future studies should be done to validate our interpretation.

In summary, with a relatively large sample of healthy young adults and a voxel-wise whole-brain analysis, we found completely different nonlinear modulation patterns of the dopamine system on the FCDs of the different functional systems, suggesting a functional system-dependent modulation of dopamine signaling. Our findings suggest that the modulation of the dopamine system on brain functions is rather complex and is influenced by many factors, such as functional networks, functional measures, and populations.

\section{References}

Aguilera M, Barrantes-Vidal N, Arias B, Moya J, Villa H, Ibáñez MI, Ruipérez MA, Ortet G, Fañanás L (2008) Putative role of the COMT gene polymorphism (Val158Met) on verbal working memory functioning in a healthy population. Am J Med Genet B Neuropsychiatr Genet 147B:898902. CrossRef Medline

Akil M, Kolachana BS, Rothmond DA, Hyde TM, Weinberger DR, Kleinman JE (2003) Catechol-O-methyltransferase genotype and dopamine regulation in the human brain. J Neurosci 23:2008-2013. Medline

Apud JA, Mattay V, Chen J, Kolachana BS, Callicott JH, Rasetti R, Alce G, Iudicello JE, Akbar N, Egan MF, Goldberg TE, Weinberger DR (2007) Tolcapone improves cognition and cortical information processing in normal human subjects. Neuropsychopharmacology 32:1011-1020. CrossRef Medline

Beck AT, Steer RA (1993) Manual for the Beck Depression Inventory. San Antonio, TX: Psychological Corporation.

Bertolino A, Fazio L, Di Giorgio A, Blasi G, Romano R, Taurisano P, Caforio G, Sinibaldi L, Ursini G, Popolizio T, Tirotta E, Papp A, Dallapiccola B, Borrelli E, Sadee W (2009a) Genetically determined interaction between the dopamine transporter and the D2 receptor on prefronto-striatal activity and volume in humans. J Neurosci 29:1224-1234. CrossRef Medline

Bertolino A, Fazio L, Caforio G, Blasi G, Rampino A, Romano R, Di Giorgio A, Taurisano P, Papp A, Pinsonneault J, Wang D, Nardini M, Popolizio T, Sadee W (2009b) Functional variants of the dopamine receptor D2 gene modulate prefronto-striatal phenotypes in schizophrenia. Brain 132:417425. CrossRef Medline

BüchelC, Friston KJ (1997) Modulation of connectivity in visual pathways by attention: cortical interactions evaluated with structural equation modelling and fMRI. Cereb Cortex 7:768-778. CrossRef Medline

Cloninger CR, Svrakic DM, Przybeck TR (1993) A psychobiological model 
of temperament and character. Arch Gen Psychiatry 50:975-990. CrossRef Medline

Cohen MX, Krohn-Grimberghe A, Elger CE, Weber B (2007) Dopamine gene predicts the brain's response to dopaminergic drug. Eur J Neurosci 26:3652-3660. CrossRef Medline

Cools R, D'Esposito M (2011) Inverted-U-shaped dopamine actions on human working memory and cognitive control. Biol Psychiatry 69:e113e125. CrossRef Medline

Dang LC, O'Neil JP, Jagust WJ (2012) Genetic effects on behavior are mediated by neurotransmitters and large-scale neural networks. Neuroimage 66C:203-214. CrossRef Medline

Dosenbach NU, Fair DA, Miezin FM, Cohen AL, Wenger KK, Dosenbach RA, Fox MD, Snyder AZ, Vincent JL, Raichle ME, Schlaggar BL, Petersen SE (2007) Distinct brain networks for adaptive and stable task control in humans. Proc Natl Acad Sci U S A 104:11073-11078. CrossRef Medline

Ettinger U, Kumari V, Collier DA, Powell J, Luzi S, Michel TM, Zedomi O, Williams SC (2008) Catechol-O-methyltransferase (COMT) val158met genotype is associated with BOLD response as a function of task characteristic. Neuropsychopharmacology 33:3046-3057. CrossRef Medline

Fallon SJ, Williams-Gray CH, Barker RA, Owen AM, Hampshire A (2013) Prefrontal dopamine levels determine the balance between cognitive stability and flexibility. Cereb Cortex 23:361-369. CrossRef Medline

Fox MD, Snyder AZ, Vincent JL, Corbetta M, Van Essen DC, Raichle ME (2005) The human brain is intrinsically organized into dynamic, anticorrelated functional networks. Proc Natl Acad Sci U S A 102:9673-9678. CrossRef Medline

Fumagalli F, Racagni G, Colombo E, Riva MA (2003) BDNF gene expression is reduced in the frontal cortex of dopamine transporter knockout mice. Mol Psychiatry 8:898-899. CrossRef Medline

Giakoumaki SG, Roussos P, Bitsios P (2008) Improvement of prepulse inhibition and executive function by the COMT inhibitor tolcapone depends on COMT Val158Met polymorphism. Neuropsychopharmacology 33:3058-3068. CrossRef Medline

Goldman-Rakic PS (1998) The cortical dopamine system: role in memory and cognition. Adv Pharmacol 42:707-711. Medline

Gong Y (1989) Manual of Wechsler Memory Scale-China revised. Changsha, China: Hunan Mapping.

Granon S, Passetti F, Thomas KL, Dalley JW, Everitt BJ, Robbins TW (2000) Enhanced and impaired attentional performance after infusion of D1 dopaminergic receptor agents into rat prefrontal cortex. J Neurosci 20: 1208-1215. Medline

Heaton R (1999) Wisconsin Card Sorting Test (computer version 3 for Windows research edition). Odessa FL: Psychological Assessment Resources.

Honea R, Verchinski BA, Pezawas L, Kolachana BS, Callicott JH, Mattay VS, Weinberger DR, Meyer-Lindenberg A (2009) Impact of interacting functional variants in COMT on regional gray matter volume in human brain. Neuroimage 45:44-51. CrossRef Medline

Khan ZU, Mrzljak L, Gutierrez A, de la Calle A, Goldman-Rakic PS (1998) Prominence of the dopamine D2 short isoform in dopaminergic pathways. Proc Natl Acad Sci U S A 95:7731-7736. CrossRef Medline

Kimberg DY, D’Esposito M, Farah MJ (1997) Effects of bromocriptine on human subjects depend on working memory capacity. Neuroreport 8:3581-3585. CrossRef Medline

KüppersE, Beyer C (2001) Dopamine regulates brain-derived neurotrophic factor (BDNF) expression in cultured embryonic mouse striatal cells. Neuroreport 12:1175-1179. CrossRef Medline

Li J, Yu C, Li Y, Liu B, Liu Y, Shu N, Song M, Zhou Y, Zhu W, Li K, Jiang T (2009) COMT val158met modulates association between brain white matter architecture and IQ. Am J Med Genet B Neuropsychiatr Genet 150B:375-380. CrossRef Medline

Liu B, Song M, Li J, Liu Y, Li K, Yu C, Jiang T (2010) Prefrontal-related functional connectivities within the default network are modulated by COMT val158met in healthy young adults. J Neurosci 30:64-69. CrossRef Medline

MännistöPT, Kaakkola S (1999) Catechol-O-methyltransferase (COMT): biochemistry, molecular biology, pharmacology, and clinical efficacy of the new selective COMT inhibitors. Pharmacol Rev 51:593-628. Medline

Mattay VS, Callicott JH, Bertolino A, Heaton I, Frank JA, Coppola R, Berman KF, Goldberg TE, Weinberger DR (2000) Effects of dextroamphetamine on cognitive performance and cortical activation. Neuroimage 12:268275. CrossRef Medline
Mattay VS, Goldberg TE, Fera F, Hariri AR, Tessitore A, Egan MF, Kolachana B, Callicott JH, Weinberger DR (2003) Catechol O-methyltransferase val158-met genotype and individual variation in the brain response to amphetamine. Proc Natl Acad Sci U S A 100:6186-6191. CrossRef Medline

Mehta MA, Owen AM, Sahakian BJ, Mavaddat N, Pickard JD, Robbins TW (2000) Methylphenidate enhances working memory by modulating discrete frontal and parietal lobe regions in the human brain. J Neurosci 20:RC65(1-6). Medline

Menon V, Uddin LQ (2010) Saliency, switching, attention and control: a network model of insula function. Brain Struct Funct 214:655-667. CrossRef Medline

O’Daly OG, Joyce D, Stephan KE, Murray RM, Shergill SS (2011) Functional magnetic resonance imaging investigation of the amphetamine sensitization model of schizophrenia in healthy male volunteers. Arch Gen Psychiatry 68:545-554. CrossRef Medline

Oldfield RC (1971) The assessment and analysis of handedness: the Edinburgh inventory. Neuropsychologia 9:97-113. CrossRef Medline

Owen AM, McMillan KM, Laird AR, Bullmore E (2005) N-back working memory paradigm: a meta-analysis of normative functional neuroimaging studies. Hum Brain Mapp 25:46-59. CrossRef Medline

Palaniyappan L, Liddle PF (2012) Does the salience network play a cardinal role in psychosis? An emerging hypothesis of insular dysfunction. J Psychiatry Neurosci 37:17-27. CrossRef Medline

Posner MI, Petersen SE (1990) The attention system of the human brain. Annu Rev Neurosci 13:25-42. CrossRef Medline

Power JD, Cohen AL, Nelson SM, Wig GS, Barnes KA, Church JA, Vogel AC, Laumann TO, Miezin FM, Schlaggar BL, Petersen SE (2011) Functional network organization of the human brain. Neuron 72:665-678. CrossRef Medline

Power JD, Barnes KA, Snyder AZ, Schlaggar BL, Petersen SE (2012) Spurious but systematic correlations in functional connectivity MRI networks arise from subject motion. Neuroimage 59:2142-2154. CrossRef Medline

Power JD, Barnes KA, Snyder AZ, Schlaggar BL, Petersen SE (2013) Steps toward optimizing motion artifact removal in functional connectivity MRI; a reply to Carp. Neuroimage 76:439-441. CrossRef Medline

Qin S, Cousijn H, Rijpkema M, Luo J, Franke B, Hermans EJ, FernándezG (2012) The effect of moderate acute psychological stress on working memory-related neural activity is modulated by a genetic variation in catecholaminergic function in humans. Front Integr Neurosci 6:16. CrossRef Medline

Reuter M, Schmitz A, Corr P, Hennig J (2006) Molecular genetics support Gray's personality theory: the interaction of COMT and DRD2 polymorphisms predicts the behavioural approach system. Int J Neuropsychopharmacol 9:155-166. Medline

Robinson PD, Schutz CK, Macciardi F, White BN, Holden JJ (2001) Genetically determined low maternal serum dopamine beta-hydroxylase levels and the etiology of autism spectrum disorders. Am J Med Genet 100:3036. CrossRef Medline

Sambataro F, Fazio L, Taurisano P, Gelao B, Porcelli A, Mancini M, Sinibaldi L, Ursini G, Masellis R, Caforio G, Di Giorgio A, Niccoli-Asabella A, Popolizio T, Blasi G, Bertolino A (2013) DRD2 genotype-based variation of default mode network activity and of its relationship with striatal DAT binding. Schizophr Bull 39:206-216. CrossRef Medline

Santiago M, Matarredona ER, Granero L, Cano J, Machado A (2000) Neurotoxic relationship between dopamine and iron in the striatal dopaminergic nerve terminals. Brain Res 858:26-32. CrossRef Medline

Seamans JK, Yang CR (2004) The principal features and mechanisms of dopamine modulation in the prefrontal cortex. Prog Neurobiol 74:1-58. CrossRef Medline

Sridharan D, Levitin DJ, Menon V (2008) A critical role for the right frontoinsular cortex in switching between central-executive and default-mode networks. Proc Natl Acad Sci U S A 105:12569-12574. CrossRef Medline

Taylor WD, ZüchnerS, Payne ME, Messer DF, Doty TJ, MacFall JR, Beyer JL, Krishnan KR (2007) The COMT Val158Met polymorphism and temporal lobe morphometry in healthy adults. Psychiatry Res 155:173-177. CrossRef Medline

Thomas G, Sinville R, Sutton S, Farquar H, Hammer RP, Soper SA, Cheng YW, Barany F (2004) Capillary and microelectrophoretic separations of ligase detection reaction products produced from low-abundant point mutations in genomic DNA. Electrophoresis 25:1668-1677. CrossRef Medline 
Tomasi D, Volkow ND (2010) Functional connectivity density mapping. Proc Natl Acad Sci U SA 107:9885-9890. CrossRef Medline

Tomasi D, Volkow ND (2011a) Functional connectivity hubs in the human brain. Neuroimage 57:908-917. CrossRef Medline

Tomasi D, Volkow ND (2011b) Association between functional connectivity hubs and brain networks. Cereb Cortex 21:2003-2013. CrossRef Medline

Tomasi D, Volkow ND (2012) Gender differences in brain functional connectivity density. Hum Brain Mapp 33:849-860. CrossRef Medline

Tune L, Barta P, Wong D, Powers RE, Pearlson G, Tien AY, Wagner HN (1996) Striatal dopamine D2 receptor quantification and superior temporal gyrus: volume determination in 14 chronic schizophrenic subjects. Psychiatry Res 67:155-158. CrossRef Medline

Usiello A, Baik JH, Rougé-PontF, Picetti R, Dierich A, LeMeur M, Piazza PV, Borrelli E (2000) Distinct functions of the two isoforms of dopamine D2 receptors. Nature 408:199-203. CrossRef Medline

Vijayraghavan S, Wang M, Birnbaum SG, Williams GV, Arnsten AF (2007) Inverted- $\mathrm{U}$ dopamine $\mathrm{D} 1$ receptor actions on prefrontal neurons engaged in working memory. Nat Neurosci 10:376-384. CrossRef Medline

Wallace DL, Vytlacil JJ, Nomura EM, Gibbs SE, D'Esposito M (2011) The dopamine agonist bromocriptine differentially affects fronto-striatal functional connectivity during working memory. Front Hum Neurosci 5:32. CrossRef Medline

Williams GV, Goldman-Rakic PS (1995) Modulation of memory fields by dopamine D1 receptors in prefrontal cortex. Nature 376:572-575. CrossRef Medline

Witte AV, FlöelA (2012) Effects of COMT polymorphisms on brain function and behavior in health and disease. Brain Res Bull 88:418-428. CrossRef Medline

Xu H, Kellendonk CB, Simpson EH, Keilp JG, Bruder GE, Polan HJ, Kandel ER, Gilliam TC (2007) DRD2 C957T polymorphism interacts with the COMT Val158Met polymorphism in human working memory ability. Schizophr Res 90:104-107. CrossRef Medline

Xu M, Moratalla R, Gold LH, Hiroi N, Koob GF, Graybiel AM, Tonegawa S (1994) Dopamine D1 receptor mutant mice are deficient in striatal expression of dynorphin and in dopamine-mediated behavioral responses. Cell 79:729-742. CrossRef Medline

Yi P, Chen Z, Zhao Y, Guo J, Fu H, Zhou Y, Yu L, Li L (2009) PCR/LDR/ capillary electrophoresis for detection of single-nucleotide differences between fetal and maternal DNA in maternal plasma. Prenat Diagn 29:217222. CrossRef Medline

Zhang Y, Bertolino A, Fazio L, Blasi G, Rampino A, Romano R, Lee ML, Xiao T, Papp A, Wang D, SadéeW (2007) Polymorphisms in human dopamine D2 receptor gene affect gene expression, splicing, and neuronal activity during working memory. Proc Natl Acad Sci U S A 104:2055220557. CrossRef Medline

Zung WW (1971) A rating instrument for anxiety disorders. Psychosomatics 12:371-379. CrossRef Medline 